\title{
C-Reactive Protein and Aggression in Patients with Schizophrenia
}

\author{
Kachouchi Aymen ${ }^{1}$, Zemrani Yassin ${ }^{2,}$, , Ahroui Yassine ${ }^{2}$, Salim Said ${ }^{1}$, Berghalout Mohammed ${ }^{1}$, \\ Adali Imane ${ }^{1}$, Manoudi Fatiha ${ }^{1}$, Chabaa Laila ${ }^{2}$, Asri Fatima ${ }^{1}$ \\ ${ }^{1}$ Research Team for Mental Health, Department of Psychiatry Marrakech, CHU Marrakech, Morocco \\ ${ }^{2}$ Biochemestry, Laboratory Arrazi, CHU Marrakech, Morocco
}

Email address:

yassinzemrani@gmail.com (Z. Yassin)

*Corresponding author

\section{To cite this article:}

Kachouchi Aymen, Zemrani Yassin, Ahroui Yassine, Salim Said, Berghalout Mohammed, Adali Imane, Manoudi Fatiha, Chabaa Laila, Asri Fatima. C-Reactive Protein and Aggression in Patients with Schizophrenia. International Journal of Psychological and Brain Sciences. Vol. 4, No. 2, 2019, pp. 7-12. doi: 10.11648/j.jpbs.20190402.11

Received: April 24, 2019; Accepted: May 24, 2019; Published: June 10, 2019

\begin{abstract}
Recent studies reported firstly an association between aggression and inflammation and secondly an association between inflammation and schizophrenia. In this study, we aim to explore the association between the clinical signs of schizophrenia, aggressive behavior in patients with relapsing schizophrenia and inflammatory marker: CRP (C-reactive protein). Adult schizophrenia inpatients without affective symptoms $(\mathrm{n}=154)$ were prospectively identified and categorized according to their C-reactive protein measurement at admission as either elevated (CRP $>5 \mathrm{mg} / \mathrm{L} ; \mathrm{n}=52$ ) or normal (CRP $<5$ $\mathrm{mg} / \mathrm{L} ; \mathrm{n}=102$ ). The following indicators of aggression were compared: PANSS excitement component (PANSS-EC), restraints, injectable treatment and suicidal behavior during hospitalization. Univariate comparisons between elevated and normal CRP levels were performed and multivariate analysis was conducted to control for relevant covariates. Inpatients with elevated C-reactive protein displayed increased aggressive behavior compared to patients with normal CRP levels $(<5 \mathrm{mg} / \mathrm{L})$. This was manifested by increased PANSS-EC score $(p=0,0001)$, higher rates of restraint during hospitalization $(p=0,0001)$, higher use of injectable treatment $(0,0001)$ and existence of suicidal behavior $(\mathrm{p}=0,001)$. Multivariate analysis revealed that Lymphocyte count, total PANSS score, PANSS excitement score were independently associated with a CRP> $5 \mathrm{mg} / \mathrm{L}$. These results confirm the role played by inflammation and immunity in the appearance of aggressive behavior in schizophrenic patients and highlight the interest of the CRP assay at the admission of patients as an indicator of aggression in hospitalized schizophrenic patients.
\end{abstract}

Keywords: Schizophrenia, Inflammation, Aggression, CRP

\section{Introduction}

Schizophrenia is a psychiatric disorder, of prolonged and disabling evolution. Its prevalence is estimated between $0.4 \%$ to $1 \%$ and it is a public health problem on a National and International level. In Morocco, the National Survey of Prevalence of Mental Disorders in the general population aged 15 and over revealed that 5.6\% suffer from a psychotic disorder and that more than 200.000 Moroccans aged 15 and over, suffer from Schizophrenia [1].

The fact that schizophrenia would be one of the most incriminated psychiatric disorder in the acting out is comforted by several data from the scientific literature, schizophrenic subjects show twice an aggressive behavior compared to the general population according to the report of the HAS on psychiatric dangerousness [2]. The risk of violence would be increased during relapses episodes of manic excitement, hallucinatory episodes or a florid outbreak of symptoms of schizophrenia [3].

Aggressive behavior is one of the leading causes of admission to a psychiatric institution and may endanger the caregiver, the patient, and other patients in the ward, hence 
the value of studying the clinical and biological signs that accompanies this behavioral disorder. A study in Germany revealed a prevalence of aggression at 7.7\% [4]. Similar studies in Australia and New Zealand have shown a prevalence of aggressive behavior from $11.3 \%$ to $15 \%[5,6]$.

CRP is a protein produced by hepatocytes after an acute or chronic inflammatory state. It is a nonspecific marker of inflammation and is associated with several pathologies, including coronary artery disease, stroke and peripheral vascular disease [7]. A meta-analysis revealed a significant increase in CRP levels in patients with schizophrenia compared to a control population [8]. However, few publications have addressed the association between aggressive behavior in patients with relapsing schizophrenia and CRP (C-reactive protein), as a biomarker. In our study, we aim to explore this relationship, the clinical signs of schizophrenia and sociodemographic data of patients; while making the assumption that patients with high CRP blood levels would be the most aggressive.

\section{Method}

\subsection{Type of Study}

This was a cross sectional study of 154 patients with schizophrenia according to the diagnostic criteria of the fifth version of DSM [9], divided into two groups according to the CRP level.

\subsection{Population Studied and Sampling}

All schizophrenic patients who were hospitalized in the Psychiatry Department at the University Hospital in Marrakech from the first of March 2017 to the 31st of May 2017 are recruited after informed voluntary consent.

\subsection{Exclusion Criteria}

We excluded patients with schizoaffective disorder, depression, bipolar disorder, and patients with organic pathology or fever over $37.9 \mathrm{C}$ and those who were treated with antibiotic, anti-inflammatory and anti-pyretic medications.

\subsection{Variables}

Data extraction from the charts were based on predesigned form which includes socio demographic measures and clinical features.

Socio demographic factors: Information was obtained from the admission charts for each patient and contains information regarding sex, age, level of education, marital status, and family history.

Clinical, therapeutic and biological features of schizophrenia: Age of onset, duration of disease, use of licit and illicit substances, psychopathological evaluation of schizophrenia was made using the positive and negative syndrome scale (PANSS) [10], PANSS excitement component (PANSS-EC) [11, 12] and PANSS depression component [13]. Biological items: CRP results, white blood cells, neutrophils, lymphocyte cells.

The evaluation of aggressiveness was made based on four clinical criteria: the PANSS score EXCITEMENT on admission, the use of restraint, the presence of suicidal ideation or suicide attempts and the administration of injectable treatment.

The evaluation of the clinical status of the patient by the PANSS score was determined by the patient's treating psychiatrist following an interview that was conducted within 48 hours from admission as a routine practice of the inpatient unit.

\subsection{Laboratory Procedures}

Blood sampling and determination of CRP: The blood sample was taken from each patient admitted systematically within a 24 hours maximum delay after admission, in the morrning after fasting for $10 \mathrm{~h}$. CRP is part of the routine biological examination at the Department. We chose the threshold of $5 \mathrm{mg} / \mathrm{L}$ as the cutoff between the high CRP group and the normal CRP group. This threshold was determined based on data from literature and similar studies [8].

\subsection{Statistical Methods}

Statistical analysis was performed using SPSS software version 19.0. The descriptive analysis consisted on a calculation of the absolute and relative frequencies for the qualitative variables, and of the positioning and dispersion parameters for the quantitative variables (mean, standard deviation). The normal distribution of variables was studied by the Kolmogorov-Smirnov test. In a bivariate analysis, the comparison of qualitative variables used the Pearson Chi2 statistical test and Fisher's if necessary. Multivariate binary logistic regression analysis was used to eliminate confounding factors and keep only those that are independently related to inflammation. Variables whose association was significant at the $20 \%$ threshold in bivariate analysis were included in a multivariate model. The variables retained in the final model were selected using a stepwise forward method with an entry threshold of 0.2 and an exit threshold of 0.05. The Hosmer Lemeshow test was used to examine the quality of the final logistic regression model. The threshold of significance was retained for a $\mathrm{p}<0.05$.

\section{Results}

\subsection{Socio-Demographic Characteristics}

Total of 154 subjects were included in the study. The mean age was $27.98 \pm 11.34$ years with extremes ranging from 18 to 61 and consisted only of men. All patients have a history of hospitalization in a psychiatric hospital and no patient had a somatic comorbidity due to the exclusion criteria (Table 1). 
Table 1. Demographic characteristics of the study subjects.

\begin{tabular}{llll}
\hline & CRP $\leq \mathbf{5 m g} / \mathbf{L} \mathbf{~ N}=\mathbf{1 0 2}$ & $\mathbf{C R P}>\mathbf{5 m g} / \mathbf{L} \mathbf{~ N}=\mathbf{5 2}$ & p-value \\
\hline Age in years, mean (SD) & $28,53(7,985)$ & $26,9(6,46)$ & 0,12 \\
Residency & $20(19,6 \%)$ & $13(25 \%)$ & 0,44 \\
Rural, n (\%) & $82(80,4 \%)$ & $39(75 \%)$ & \\
Urban, n (\%) & $90(88,2 \%)$ & $42(80,8 \%)$ & 0,21 \\
Occupation, $\mathrm{n}(\%)$ & $12(11,8 \%)$ & $10(19,2 \%)$ & \\
Unemployed & $98(96,1 \%)$ & $52(100 \%)$ & 0,017 \\
Employed & $4(3,9 \%)$ & 0 & 0,77 \\
Education, n (\%) & $69(67,6 \%)$ & $34(65,4 \%)$ & \\
Pimary and secondary & & \\
High and university & & \\
Lower socio economic status & & \\
\hline
\end{tabular}

\subsection{CRP Levels and Clinical and Biological Features of Schizophrenia}

Table 2 shows the associations between clinical and biological characteristics of schizophrenic patients and the rate of CRP.

Table 2. Study of the association between CRP level and clinical and biological characteristics of schizophrenia.

\begin{tabular}{|c|c|c|c|}
\hline & $\mathrm{CRP} \leq 5 \mathrm{mg} / \mathrm{L} \mathrm{N}=102$ & $\mathrm{CRP}>5 \mathrm{mg} / \mathrm{L} \mathrm{N}=52$ & p-value \\
\hline Age of schizophrenia onset, mean (SD) & $22,45(3,811)$ & $21,21(2,919)$ & 0,027 \\
\hline Duration of illness, (years) mean (SD) & $6,08(6,174)$ & $5,79(4,964)$ & 0,76 \\
\hline \multicolumn{4}{|l|}{ Smoking status } \\
\hline No, n (\%) & $24(23,5 \%)$ & $4(7,7 \%)$ & \\
\hline \multicolumn{4}{|l|}{ Cannabis Use } \\
\hline Yes, $\mathrm{n}(\%)$ & $51(72,9 \%)$ & $19(27,1 \%)$ & 0,12 \\
\hline \multicolumn{4}{|l|}{ Clinical measures } \\
\hline Total PANSS, mean (SD) & $64,01(7,744)$ & $78,81(11,244)$ & 0,0001 \\
\hline PANSS positive, mean (SD) & $21,94(4,807)$ & $27,52(5,731)$ & 0,0001 \\
\hline PANSS negative, mean (SD) & $12,74(2,725)$ & $14,44(2,967)$ & 0,0001 \\
\hline PANSS general psychopathology, mean (SD) & $29,15(5,33)$ & $35,35(8,510)$ & 0,0001 \\
\hline PANSS depression, mean (SD) & $5,96(1,482)$ & $6,29(1,753)$ & 0,25 \\
\hline \multicolumn{4}{|l|}{ Laboratory parameters } \\
\hline White blood cells $\left(10^{3} / \mathrm{ml}\right)$, mean (SD) & $8,241(1,0547)$ & $8,249(1,4734)$ & 0,97 \\
\hline Lymphocyte cells $\left(10^{3} / \mathrm{ml}\right)$, mean (SD) & $1,197(0,5095)$ & $1,712(0,7635)$ & 0,0001 \\
\hline \multicolumn{4}{|l|}{ Aggression measures } \\
\hline PANSS excitement, mean (SD) & $8,76(3,591)$ & $18,33(3,833)$ & 0,0001 \\
\hline Restraint during hospitalization n (\%) & $17(16,7 \%)$ & $47(90,4 \%)$ & 0,0001 \\
\hline Suicidal ideation or suicide attempt $\mathrm{n}(\%)$ & $6(5,9 \%)$ & $13(25 \%)$ & 0,001 \\
\hline Injectable treatment, $\mathrm{n}(\%)$ & $12(11,8 \%)$ & $48(92,3 \%)$ & 0,0001 \\
\hline
\end{tabular}

\subsection{Logistic Regression}

Table 3. Binary logistic regression model with CRP> $5 \mathrm{mg} / \mathrm{L}$.

\begin{tabular}{|c|c|c|c|c|c|c|}
\hline & B & $\chi 2$ & $p$ & OR & \multicolumn{2}{|c|}{ OR (IC à 95,0\%) } \\
\hline Education level & ,978 & ,425 &, 514 & 2,660 &, 140 & 50,357 \\
\hline Substance use &, 871 & 249 & ,618 & 2,389 & ,078 & 73,056 \\
\hline Age of schizophrenia onset & $-1,788$ & 1,458 & ,227 &, 167 & ,009 & 3,047 \\
\hline Total PANSS & ,443 & 4,392 & ,036 & 1,558 & 1,029 & 2,357 \\
\hline PANSS Positive &,- 398 & 2,479 &, 115 & ,671 & ,409 & 1,102 \\
\hline PANSS Negative &,- 549 & 3,197 &, 074 &, 578 & ,316 & 1,054 \\
\hline PANSS EXC & 622 & 5,585 &, 018 & 1,863 & 1,112 & 3,121 \\
\hline Restraint during hospitalization & $-2,348$ & 1,183 & 277 & 096 & 001 & 6,579 \\
\hline Suicidal ideation or suicide attempt &,- 497 & ,064 &, 801 & ,609 &, 013 & 28,902 \\
\hline Injectable treatment & $-1,800$ & ,690 & ,406 &, 165 &, 002 & 11,545 \\
\hline Constante & $-8,794$ & 1,432 & ,231 &, 000 & & \\
\hline
\end{tabular}

$\beta$ : Bêta constante, $\chi 2$ : Wald, $\mathrm{p}$ : the threshold of significance for the Wald test, OR: Odds Ratio. 
A binary logistic regression of CRP $>5 \mathrm{mg} / \mathrm{L}$ was performed to evaluate the coefficients of variation (Table 3 ). We found that the variables: Lymphocyte count, total PANSS score, PANSS excitement score were independently associated with a CRP $>5$ $\mathrm{mg} / \mathrm{L}$.

\section{Discussion}

Our study aimed to confirm the hypothesis that hospitalized patients with schizophrenia with elevated CRP would be more aggressive.

We chose in our study four clinical parameters as indicators of aggressive behavior in schizophrenic patients:

PANSS Score Excitement, use of physical restraint during hospitalization, presence of suicidal ideation or suicide attempts, and administration of injectable medications. As a result, we found a positive correlation between the four aggression indicators and the increase in CRP.

\subsection{Panss Exc}

In our study, the PANSS EXC score was 10 points higher in the high CRP group compared to the other group. Patients with high CRP required five times more the use of mechanical restraint. These two results are consistent with the Barzilay study, which retrospectively compared 213 schizophrenic patients with elevated CRP with 156 schizophrenic patients with normal CRP. This study found a positive relationship between the PANSS EXC score and the high CRP score, the score was two points higher and the use of restraint was multiplied by 2.48 in the high CRP group [14].

Another study that assessed the clinical and biological characteristics of agitated schizophrenic patients $(\mathrm{N}=32)$ compared to a healthy control group $(\mathrm{N}=42)$ concluded that the patients with elevated CRP levels have more severe clinical symptoms [15]. This difference could be explained by several factors: the delay of care and demand for care, the complexity on the access to psychiatric care, poor family care and especially in our study by the presence of substance abuse.

\subsection{Injectable Treatment and Use Physical of Restraint}

The administration of injectable treatment was present in $92.5 \%$ of patients with a high CRP, concord with the use of physical restraint. These results ask the efficiency of communication techniques and the quality of the therapeutic alliance of carers vis-à-vis these patients.

\subsection{Suicidal Ideation and Suicide Attempts}

The presence of suicidal ideation or suicide attempts was five times higher in schizophrenic patients with high CRP. Several studies revealed the association of an inflammatory state characterized by the elevation of inflammatory markers at central and peripheral level with suicidal ideation and suicidal act $[16,17]$. However, suicidality requires the use of specific scales as well as an analysis of the context and meaning of the act during the suicide attempt to have a better understand this pattern.

\subsection{CRP and Psychopathological Signs of Schizophrenia}

We found that patients with elevated CRP had a total PANSS score and scores of subscales: PANSS positive and negative significantly elevated compared to the normal CRP group. The exploration of psychopathological signs of schizophrenia in relation to biological markers was subject to some studies with controversial results [18]. Thus, Fan and all showed a significant rise in PANSS scores and PANSS subscale scores except for positive PANSS by comparing two groups of patients with schizophrenia according to the CRP rate [19]. Although In an Egyptian study of 200 patients with schizophrenia after CRP measurement and PANSS clinical assessment, a CRP elevation was accompanied by a high score of total PANSS and PANSS sub-scales [20]. Nonthelesse Joseph and all study of 88 patients found no significant relationship between CRP and clinical signs of schizophrenia [21].

Otherwise, Dickerson and all found that high CRP levels were related to greater cognitive impairment and did not find a relationship between $\mathrm{CRP}$ and the severity of psychopathological symptoms [22]. Similarly, in Kuwaiti study of 207 patients, CRP was not significantly associated with the severity of clinical signs, but with the presence of catatonic syndrome [23].

\subsection{CRP and Depression in Patients with Schizophrenia}

Our study found no significant relationship between the PANSS depression score and the CRP level and no patients in both groups were on antidepressants. This result consists with the Barzilay and all study with an average of 8.77 for the scale score and an average of 6.07 for our study [14]. However, several studies have highlighted the elevation of inflammation markers in depressed patients with suicidal ideation $[16,17]$. The Valkanova and all meta-analysis which included eight studies for CRP (14832 participants) and three studies for IL-6 (3695 participants) showed a significant association between the elevation of these two biological markers, especially CRP with the presence depression and the severity of depressive symptoms [24]. This result should be interpreted with caution as the PANSS depression items could be confused with the negative symptoms of schizophrenia or the side effects of neuroleptics. It would be better to combine two or three scales of depression for more reliable results.

\subsection{CRP and the Other Biological Parameters}

For the other biological parameters, we did not find any significant difference between the two groups concerning the number of white blood cells, whereas a very significant association was found between the lymphocyte number and the CRP level, thus the Lymphocyte count was 
independently associated with CRP $>5 \mathrm{mg} / \mathrm{L}$ with $\mathrm{RRs}$ estimated by the following Odds Ratio (29.45, 95\% CI: 2.08 and 416.32). The study by Pan and all found a significant difference between the number of white blood cells and the CRP level without evaluation of the number of lymphocytes [15]. The study of Barzilay and all studied several biological parameters and found a positive correlation between CRP and white blood cell count, lymphocyte cell count, neutrophil count, albumin and platelet count [14]. However, other studies did not reproduce the same results $[19,20]$. The meta-analysis of Miller and all provides more clarification and proposes that CD4, CD8, and CD4 / CD8 significantly increase during a first psychotic episode, that the ratio CD4 / CD8 appears as a state marker seen that it is normalized after treatment with neuroleptics while the CD56 would be a marker trait since it remains high even after treatment [25].

\subsection{Perspectives}

Current research in psychiatry is increasingly interested in biological parameters in search of a possible links between clinical signs and biological indicators, the study we conducted to our knowledge would be the first in Morocco that treats inflammation, markers and schizophrenia and among the few that have focused specifically on the aggressive behavior of relapsing schizophrenic patients in relation to a biological marker. These results would be interesting to the extent of a reflection around a staging of schizophrenic patients according to the likely risk of aggression and acting out, and thus to adapt the medical management of each patient according to his potential to violence. Indeed recent studies have relied in the presence of inflammatory mechanisms to propose new therapeutic classes in combination with neuroleptics, Muller and all reported that the use of anti-inflammatory drugs steroids Celexocib in combination with risperidone for 5 weeks for the treatment of patients with relapsing-reactive schizophrenia can improve the PANSS score [26-29], other studies with the same molecule or with aspirin showed the same results [30, 31]. On the other hand, the study of Rapaport and Muller with Celexocib and two clinical trials with minocycline found an effect limited to the acute phase and which is not reproducible and not durable [32-34]. These therapeutic data confirm the importance of stratifying and codifying the management of patients according to the institutional or ambulatory framework, the clinical and biological profile of schizophrenic patients.

\subsection{Limitations}

There are limits to our research: the absence of female schizophrenic patients, we can not be certain that the elevation of CRP is specific to the aggression of schizophrenic patients, it could be a common phenomenon of relapse or the acute phase regardless the initial psychiatric pathology.

\section{Conclusion}

Schizophrenia remains in our context the most frequent psychiatric disorder at the hospital level with a long history of stigmatization based on behavioral disorders, violence and hetero-aggressive behavior that may exist during the evolution of the disease and at the time of relapses. Our study highlighted several findings: First, hospitalized patients with schizophrenia who have a high CRP have significantly more aggressive behaviors. Second, the CRP level is also correlated with the severity of the psychopathological symptoms assessed by the PANSS scale. Third, the number of lymphocyte cells significantly follows the rise in CRP levels. Fourthly, the predictive factors for CRP elevation in schizophrenic patients after logistic regression are: the total PANSS score, the general PANSS score, and the number of lymphocyte cells.

Thus, these results confirm the role played by inflammation and immunity in the appearance of aggressive behavior in schizophrenic patients and highlight the interest of the CRP assay at the admission of patients as an indicator of aggression in hospitalized patients with schizophrenia.

\section{Conflicts of Interest}

The authors declare that they have no competing interest.

\section{References}

[1] Ministère de la santé marocain. Enquête nationale sur les prévalences des troubles mentaux en population générale, (ENPTM 2003-2006).

[2] Haute autorité de santé HAS: Dangerosité psychiatrique: étude et évaluation des facteurs de risque de violence hétéroagressive chez les personnes ayant des troubles schizophréniques ou des troubles de l'humeur (2011).

[3] Volavka J, Laska E, Baker S, Meisner M, Czobor P, Krivelevich I. History of violent behaviour and schizophrenia in different cultures. Analyses based on the WHO study on determinants of outcome of severe mental disorders. Br. J. Psychiatry. 1997; 171, 9-14.

[4] Ketelsen R, Zechert C, Driessen M, Schulz M. Characteristics of aggression in a German psychiatric Hopsital and predictors of patients at risk. J. Psychiatr. Ment. Health. Nurs. 2007; 14:92-99.

[5] El-Badri, S. M., Mellsop, G. Aggressive behaviour in an acute general adult psychiatric unit. Psychiatr. Bull. 2006; 30: 166168.

[6] Carr V. J, Lewin T. J, Sly K. A, Conrad A. M, Tirupati S, Cohen $\mathrm{M}$ and all. Adverse incidents in acute psychiatric inpatient units: rates, correlates and pressures. Aust. N. Z. J Psychiatry. 2008; 42 (4): 267-282.

[7] Ridker P. M. C-Reactive Protein A Simple Test to Help Predict Risk of Heart Attack and Stroke. Circulation. 2003; 108 (12): 81-85. 
[8] Miller B. J, Culpepper N, Rapaport, M. H. C-reactive protein levels in schizophrenia: a review and meta-analysis. Clin. Schizophr. Relat. Psychoses. 2014; 7 (4): 223-230.

[9] APA. Diagnostic and statistical manual of mental disorders. $5^{\mathrm{h}}$ ED. Washington DC: American Psychiatric Association; (2014).

[10] Kay S. R, Fiszbein A, Opler L. A. The positive and negative syndrome scale (PANSS) for schizophrenia. Schizophr. Bull. 1987; 13 (2): 261-276.

[11] Lindenmayer J-P, Brown E, Baker R. W, Schuh L. M, Shao L, Tohen M, and all. An excitement subscale of the Positive and Negative Syndrome Scale. Schizophr. Res. 2014; 68: 331-7.

[12] Montoya A, Valladares A, Lizan L, San L, Escobar R, Paz S. Validation of the Excited Component of the Positive and Negative Syndrome Scale (PANSS-EC) in a naturalistic sample of 278 patients with acute psychosis and agitation in a psychiatric emergency room. Health. Qual. Life. Outcomes. 2011; 9:18.

[13] El Yazaji M, Battas O, Agoub M, Moussaoui D, Gutknecht C, Dalery $\mathrm{J}$ and all. Validity of the depressive dimension extracted from principal component analysis of the PANSS in drug-free patients with schizophrenia. Schizophr. Res. 2002; 56 (1-2): 121- 7.

[14] Barzilay R, Lobel T, Krivoy A, Shlosberg D, Weizman A, Katz N. Elevated C-reactive protein levels in schizophreniain patients is associated with aggressive behavior. Eur. Psychiatry. 2016: 31: 8-12.

[15] Pan S, Tan Y, Yao S, Zhao X, Xiaong J. Serum highsensitivity C-reactive protein: A delicate sentinel elevated in drug-free acutely agitated patients with schizophrenia. Psychiatry. Res. 2016; 246:89-94.

[16] Lindqvist D, Janelidze S, Hagell P, Erhardt S, Samuelsson M, Minthom L and all. Interleukin-6 is elevated in the cerebrospinal fluid of suicide attempters and related to symptom severity. Biol. Psychiatry. 2009; 66 (3): 287-92.

[17] Janelidze S, Mattei D, Westrin A, Traskman-Bendz L, Brundin L. Cytokine levels in the blood may distinguish suicide attempters from depressed patients. Brain. Behav Immun. 2011; 25 (2): 335-9.

[18] Solanki R. K, Singh P, Singh M, Sinha M, Swami M. K, Saini S. C-reactive protein (CRP) in patients with schizophrenia: are they related with symptomatology? J. Ment Heal. Hum. Behav. 2009; 15 (1): 6-10.

[19] Fan X, Pristach C, Liu E. Y, Freudenreich O, Henderson D. C, Goff D. C. Elevated serum levels of C-reactive protein are associated with more severe psychopathology in a subgroup of patients with schizophrenia. Psychiatry. Res. 2007; 149:26771.

[20] Fawzi M. H, Fawzi M. M, Fawzi M. M, Said N. S. C-reactive protein serum level in drug-free male Egyptian patients with schizophrenia. Psychiatry. Res. 2011; 190: 91-7.

[21] Joseph J, Depp C, Sirkin M, Daly R, Glorioso D, Palmer B, and all. Associations of High Sensitivity C-Reactive Protein Levels in Schizophrenia and Comparison Groups. Schizophr. Res. 2015; 168 (0): 456-460.
[22] Dickerson F, Stallings C, Origoni A, Boronow J, Yolken R. Creactive protein is associated with the severity of cognitive impairment but not of psychiatric symptoms in individuals with schizophrenia. 2007; 93 (1-3):261-5.

[23] Akanji A. O, Ohaeri J. U, Al-Shammri S, Fatania H. R. Association of blood levels of C- reactive protein with clinical phenotypes in Arab schizophrenic patients. Psychiatry. Res. Aug. 2009; 169 (1): 56-61.

[24] Valkanova V, Ebmeier K. P, Allan C. L. CRP, IL-6 and depression: a systematic review and meta-analysis of longitudinal studies. J. Affect. Disord. 2013; 150 (3): 736-44.

[25] Miller B. J, Gassama B, Sebastian D, Buckley P, Mellor A. Meta-Analysis of Lymphocytes in Schizophrenia: Clinical Status and Antipsychotic Effects. Biol. Psychiatry. 2013; 73 (10): 993-999.

[26] Muller N, Riedel M, Scheppach C, Brandstatter B, Sokullu S, Krampe K, and all. Beneficial antipsychotic effects of celecoxib add-on therapy compared to risperidone alone in schizophrenia. Am. J. Psychiatry. 2002; 159 (6):1029-34.

[27] Muller N, Ulmschneider M, Scheppach C, Schwarz M. J, Ackenheil M, Moller H. j., and all. COX-2 inhibition as a treatment approach in schizophrenia: Immunological considerations and clinical effects of celecoxib add-on therapy. Eur. Arch. Psychiatry. Clin. Neurosci. 2004; 254 (1):14-22.

[28] Muller N. Inflammation and the glutamate system in schizophrenia: implications for therapeutic targets and drug development. Expert. Opin. Ther. Targets. 2008; 12 (12): 1497- 507.

[29] Muller N, Krause D, Dehning S, Musil R, Schennach-Wolff R, Obermeier M and all. Celecoxib treatment in an early stage of schizophrenia: results of a randomized, doubleblind, placebocontrolled trial of celecoxib augmentation of amisulpride treatment. Schizophr. Res. 2010; 121 (1-3):118-124.

[30] Akhondzadeh S, Tabatabaee M, Amini H, Ahmadi Abhari S. A, Abbasi S. H, Behnam, B. Celecoxib as adjunctive therapy in schizophrenia: a double-blind, randomized and placebo controlled trial. Schizophr. Res. 2007; Feb 90 (1-3): 179-85.

[31] Laan W, Grobbee D. E, Selten J-P, Heijnen C. J, Kahn R. S, Burger H. Adjuvant aspirin therapy reduces symptoms of schizophrenia spectrum disorders: results from a randomized, double blind, placebo-controlled trial. J. Clin. Psychiatry. 2010; 71:520-7.

[32] Rapaport M, Müller N. Immunological states associated with schizophrenia. Psychoneuroimmunology. 2001; 2: 373-382.

[33] Levkovitz Y, Mendlovich S, Riwkes S, Braw Y, LevkovitchVerbin H, Gal G, and all. A double-blind, randomized study of minocycline for the treatment of negative and cognitive symptoms in early-phase schizophrenia. J. Clin. Psychiatry. 2010; 71 (2):138-149.

[34] Miyaoka T, Yasukawa R, Yasuda H, Hayashida M, Inagaki T, Horiguchi J. Minocycline as adjunctive therapy for schizophrenia: an open-label study. Clin. Neuropharmacol. 2008; 31:287-292. 